\title{
Health outcomes, education, healthcare delivery and quality - 3040. Occurrence and effects of nasal polyps in patients with bronchial asthma and/or allergic rhinitis
}

\author{
Swati Behera \\ From 2nd WAO International Scientific Conference (WISC 2012) \\ Hyderabad, India. 6-9 December 2012
}

\section{Background}

The occurrence of nasal polyps (NP) in bronchial asthma (BA) and/or allergic rhinitis (AR) remains largely undiagnosed and adversely affects quality of life (QoL).

\section{Methods}

The study comprised 220 consecutive patients (males/ females, 123/97), 15 to 60 years with BA and/or AR enrolled from outpatients department of VP Chest Institute, University of Delhi. BA/AR was diagnosed according to GINA/ARIA guidelines respectively. Group1 comprised 35 patients with BA, group2, 43 patients with AR and group3, 142 patients with both diseases. CT-PNS, done in all patients, assessed CRS/NP and was scored with Lund Mackey Score (LMS). To assess the effect of NP, asthmatics responded to asthma QoL questionnaire (MiniAQLQ), and the 15-17 years to MiniPAQLQ. Rhinitics responded to rhinoconjunctivitis QoL questionnaire (RQLQ) and the 15-17 years to AdolRQLQ. Patients with nasal symptoms responded to Sinonasal Outcome Test 22 (SNOT 22) and Visual Analogue Scale (VAS).

\section{Results}

Of the 220 patients, $190(86.4 \%)$ had CRS. Of these, 138 (72.6\%) had NP. CRS was seen in 26/35 (74.3\%) patients in group1, 38/43(88.4\%), in group2 and 126/142 (88.7\%), in group3. NP was seen in 18/26 (69.2\%), 30/38 (78.9\%) and $90 / 126(71.4 \%)$ in groups 1,2 and 3 respectively. The presence of NP increased mean LMS score from 5 in CRS to 8 $(P=0.005)$ in $\mathrm{CRS} / \mathrm{NP}$ patients. In group2, occurrence of NP increased mean Global VAS score from 5 to $7(P=0.029)$,
SNOT 22 scores from 43 to $44(P=0.029)$ and in RQLQ scores, activities score rose from 1.7 to $3.1(P=0.007)$, nasal symptom score from 3.9 to $4.1(P=0.033)$, non hayfever symptom score from 2.3 to $3.5(P=0.045)$ and RQLQ overall scores from 2.4 to $3.1(P=0.023)$. In group3, NP increased mean SNOT 22 scores from 39 to $42(P=0.002)$, RQLQ nasal symptom score from 4.2 to $4.4(P=0.001)$ and RQLQ troubled sleep score from 1.7 to $1.9(P=0.021)$. There were no significant differences in group1. In all three groups, presence of NP increased mean RQLQ activities score from 3 to $3.3(P=0.033)$ and RQLQ troubled sleep score from 1.7 to $2(P=0.010)$.

\section{Conclusions}

NP was seen in nearly two-third (62.7\%) of patients with BA and/or AR. QoL was maximally impaired when AR was complicated with NP followed by patients with both diseases.

Published: 23 April 2013

doi:10.1186/1939-4551-6-S1-P213

Cite this article as: Behera: Health outcomes, education, healthcare delivery and quality - 3040. Occurrence and effects of nasal polyps in patients with bronchial asthma and/or allergic rhinitis. World Allergy Organization Journal 2013 6(Suppl 1):P213. 